Technological University Dublin

DÜBLIN

ARROW@TU Dublin

2018

\title{
Measurements of Milli-Newton Surface Tension Forces with Tilted Fiber Bragg Gratings
}

\author{
Changyu Shen \\ Technological University Dublin \\ Chuan Zhong \\ Trinity College Dublin \\ Dejun Liu \\ Technological University Dublin, dejun.liu@tudublin.ie
}

See next page for additional authors

Follow this and additional works at: https://arrow.tudublin.ie/engscheleart2

Part of the Engineering Commons

\section{Recommended Citation}

Shen, C., Zhong, C. \& Liu, D. (2018). Measurements of milli-Newton surface tension forces with tilted fiber Bragg gratings. Optic Letters, vol 43, no. 2, pp. 252-258. doi.org/10.1364/OL.43.000255.

This Article is brought to you for free and open access by the School of Electrical and Electronic Engineering at ARROW@TU Dublin. It has been accepted for inclusion in Articles by an authorized administrator of ARROW@TU Dublin. For more information, please contact arrow.admin@tudublin.ie, aisling.coyne@tudublin.ie, gerard.connolly@tudublin.ie.

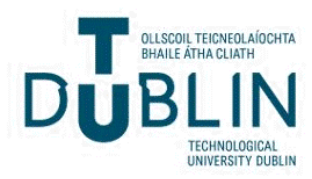




\section{Authors}

Changyu Shen, Chuan Zhong, Dejun Liu, Xiokang Lian, Jianyao Zheng, Jingjing Wang, Yuliya Semenova, Gerald Farrell, Jacques Albert, and John F. Donegan 


\title{
Measurements of milli-Newton surface tension forces with tilted fiber Bragg gratings
}

\author{
Changyu Shen, ${ }^{1,2,3,5,6, *}$ Chuan Zhong, ${ }^{2}$ Dejun Liu, ${ }^{3}$ Xiaokang Lian, ${ }^{3}$ Jianyao Zheng, ${ }^{2}$ Jinguing Wang, ${ }^{6}$ \\ Yuliya Semenova, ${ }^{3,7}$ Gerald Farrell, ${ }^{3,8}$ Jacques Albert, $^{4}$ and John F. Donegan ${ }^{2,6,9}$ \\ ${ }^{1}$ Institute of Optoelectronic Technology, China Jiliang University, Hangzhou 310018, China \\ ${ }^{2}$ School of Physics, Trinity College Dublin, Dublin 2, Ireland \\ ${ }^{3}$ Photonics Research Center, Dublin Institute of Technology, Kevin Street, Dublin 8, Ireland \\ ${ }^{4}$ Department of Electronics, Carleton University, Ottawa, Ontario K1S 5B6, Canada \\ ${ }^{5}$ State Key Laboratory of Optical Instrumentation, Zhejiang University, Hangzhou 310027, China \\ ${ }^{6}$ Centre for Research on Adaptive Nanostructures and Nanodevices (CRANN), Trinity College Dublin, Dublin 2, Ireland \\ 7e-mail: yuliya.semenova@dit.ie \\ ${ }^{8}$ e-mail: gerald.farrell@dit.ie \\ ${ }^{9} e$-mail: jdonegan@tcd.ie \\ *Corresponding author: shenchangyu@cjlu.edu.cn
}

Received 9 October 2017; revised 1 December 2017; accepted 3 December 2017; posted 7 December 2017 (Doc. ID 308747); published 9 January 2018

Small lateral forces (lower than $0.1 \mathrm{~N}$ ) cannot normally be measured with conventional single-mode fiber-based sensors because of the high value of their Young modulus (>70 GPa). Here we demonstrate the measurement of lateral forces in the range from 0.2 to $1.4 \times 10^{-3} \mathrm{~N}$ with a tilted fiber Bragg grating (TFBG) in conventional single-mode fiber pushed against the surface tension (ST) of a bead of water. The measured transmission changes of individual cladding mode resonances of the TFBG corresponding to these force values are of the order of $29 \mathrm{~dB}$. Separate measurements of the contact angle between the surface of the water and the fiber are used to calibrate the sensor with help from the known value of ST for water. Once calibrated, a TFBG can be used to measure unknown forces in the same range or to measure an unknown ST, provided a separate force measurement is available. (c) 2018 Optical Society of America

OCIS codes: (060.3735) Fiber Bragg gratings; (060.2370) Fiber optics sensors.

https://doi.org/10.1364/OL.43.000255

Liquid molecules situated near or at the interface (i.e., liquidgas or liquid-solid) have different spatial orientations with respect to each other, compared to the molecules in the bulk phase [1-4]. The intermolecular attraction forces acting on each molecule in the bulk liquid phase are isotropic at a fixed temperature, and this means that the resultant force in any direction is balanced. The molecules at the interface, however, experience an asymmetrical force field, which results in a so-called surface tension (ST) for liquid-vapor interfaces or interfacial tension, for interfaces between two liquids, two solids, or a liquid and a solid. ST and its molecular dynamics at the interface are important in a variety of areas, such as engineering, biochemistry, electrochemistry, and chromatography. Many studies have been reported on contact angle measurements of ST, super hydrophobic materials, ST dynamics, and so on [5-10], but, to the best of our knowledge, there have been no reports on the effect of ST on optical fibers. This is likely due to the fact that typical ST forces are in the $10^{-3} \mathrm{~N}$ range, i.e., much too small to be detectable by optical fiber force sensors with a Young's modulus above $70 \mathrm{GPa}$ (for silica fibers) [11-13]. A possible method to overcome this drawback would be to reduce the fiber diameter to increase the force sensitivity, such as in microfiber Bragg gratings [14-16] and microfiber-based asymmetrical Fabry-Perot interferometers [17]. However, one problem is that reducing the fiber diameter makes the sensors easy to break; another problem is that such force sensors [14-17] are not designed to measure lateral forces, but rather axial ones. On the other hand, there is no way to measure the ST of an unknown liquid (just knowing the liquid's refractive index (RI) in the range of 1.33-1.43, for instance) by using the water as a reference.

Here we demonstrate that a standard single-mode fiberbased tilted fiber Bragg grating (TFBG) can be used as a sensor for measuring $\mathrm{mN}$-level forces exerted by the ST of liquids. The TFBG is made in physically unmodified fiber in which a photoinduced grating is inscribed and where the tilt of the grating planes relative to the fiber axis allows for the excitation of multiple cladding propagating modes, whose evanescent field outside the cladding surface can be used for sensing [18-22]. The measurement principle for small lateral forces is based on the change in the contact angle between a liquid surface and the optical fiber, as it is "pushed" against a free-standing liquid bead. The gradual change in fiber surrounding results in a change in the distribution of cladding modes excited by the TFBG. This method can be applied to any liquid with a RI in the range from 1.33 to 1.44 because, under that RI range, the spectral responses of the TFBG are similar to that of water. 


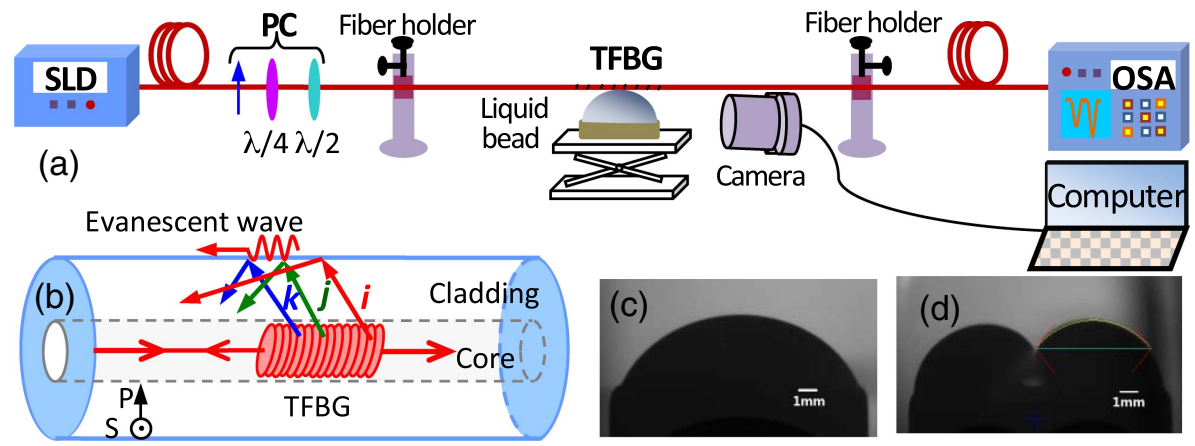

Fig. 1. Liquid ST sensing principle with TFBG. (a) Experimental setup used to interrogate the sensor. (b) Coupling of core-guided light into a set of cladding modes by the TFBG. $S$ and P indicate the polarization direction relative to the fiber. (c) Camera image of the liquid bead before the TFBG thrust into it. (d) Camera image of the liquid bead during the TFBG thrust into it; the water bead was divided by the fiber into two symmetrical halves with a contact angle of $50.62^{\circ}$.

Figure 1(a) illustrates a schematic diagram of the TFBGbased liquid ST sensing system. A fiber-coupled superluminescent diode source (THORLABS), polarization controller (PC), and optical spectrum analyzer (OSA) (Agilent, 86142B) were used to record the transmission spectra of the TFBG with P and $S$ linearly polarized input light relative to the direction of the tilt of the grating planes [18]. The spectral resolution of the OSA is $0.06 \mathrm{~nm}$. The PC is made up of a polarizer, a half-wave plate, and quarter-wave plate, thus allowing the generation of arbitrary polarization states at the input of the fiber, which can compensate for any change of polarization state induced by fiber loops and twists between the PC and the TFBG. While measurements with linearly polarized light are not strictly necessary in these experiments, they provide narrower resonances, since each resonance of a TFBG includes contributions from several kinds of hybrid modes of different polarizations (TE, $\mathrm{TM}, \mathrm{HE}$, and $\mathrm{EH}$ ) that are slightly separated in wavelength and, thus, cause broadening of resonances in unpolarized measurements [18]. For the measurements of ST, the TFBG was fixed rigidly between fiber holders in such a manner that its whole length was in contact with the liquid bead. The fiber and the liquid bead were placed on three-dimensional translation stages that allowed for the TFBG to be lowered gradually into the liquid. The camera system is used simultaneously to record and calculate the contact angle between the fiber and the liquid bead with an image processing system. The contact angle was determined by using the "telescope-goniometer" method which is a direct measurement of the tangent angle at the three-phase contact point using a contact angle analyzer (First Ten Angstroms Inc., Portsmouth, VA).

Figure 1(b) illustrates the coupling of core-guided light into a set of cladding modes by the TFBG, whereas these cladding modes have an evanescent surface wave propagating outside the cladding that can interact with the surrounding medium (air and water, in various proportions depending on the contact angle) [18]. Finally, Figs. 1(c) and 1(d) show camera images of the liquid bead before and during the TFBG thrust into it, respectively. In the latter case, the water bead was divided by the fiber into two symmetrical halves with a contact angle of $50.62^{\circ}$.

Corning SMF-28 fiber was used to fabricate the TFBG. The fiber was kept in a hydrogen chamber for 15 days at 2500 psi and room temperature $\left(25^{\circ} \mathrm{C}\right)$ to improve its photosensitivity. A $1 \mathrm{~cm}$ long TFBG with a tilt angle of $8^{\circ}$ and a period of
$542 \mathrm{~nm}$ was inscribed in the hydrogen-loaded fiber using an argon ion laser and the phase mask technique. The length of the TFBG is chosen so that the resonances in air and water are narrow enough to avoid overlapping and have deep transmission minima. The angle is chosen so that high-order cladding modes (most sensitive to external indices near that of water) have strong resonances.

The standard phase-matching condition for the resonance wavelength of the core mode $\lambda_{\text {Bragg }}$ and of any $i$ th cladding mode $\lambda_{\text {clad }}^{i}$ in the TFBG transmission spectrum can be expressed as [18]

$$
\begin{gathered}
\lambda_{\text {Bragg }}=2 n_{\mathrm{eff}}(\text { core }) * \frac{\Lambda}{\cos (\theta)}, \\
\lambda_{\text {clad }}^{i}=\left(n_{\mathrm{eff}}(\text { core })+n_{\mathrm{eff}}^{i}(\mathrm{clad})\right) * \Lambda / \cos (\theta),
\end{gathered}
$$

where $n_{\text {eff(core) }}$ and $n_{\text {eff }}^{i}$ (clad) are the effective indices of the fiber core mode and cladding mode, respectively. $\Lambda$ is the grating period measured perpendicular to the grating planes, and $\theta$ is the tilt angle of the grating planes. As shown in the transmission spectrum of such a grating shown in Fig. 2, the grating couples the core mode to a multitude of cladding modes (each

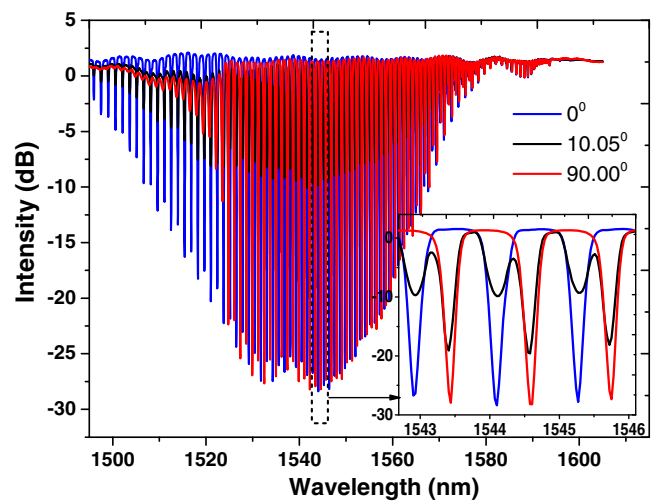

Fig. 2. P-polarized transmitted power spectra of a $10 \mathrm{~mm}$ long $8^{\circ}$ TFBG in air (blue curve, labeled $0^{\circ}$ ), in water (red curve, labeled $90^{\circ}$ ), and partially being pushed into the water with a contact angle of $10.05^{\circ}$ (black curve, labeled $10.05^{\circ}$ ). The inset shows the detailed spectra of the above three labeled curves at the wavelength range of $1542-1546 \mathrm{~nm}$. 
at a different wavelength, as determined by the grating period and mode effective index), and these couplings show up as loss peaks in the transmission spectrum.

In Fig. 2, the spectra of the TFBG in air, completely immersed in water, and partially pushed into the water bead with a contact angle of $10.05^{\circ}$ are presented. It can be seen from the inset of Fig. 2 that as the TFBG is being forced into the water bead, an individual cladding mode resonance gets split in two, at wavelengths corresponding to the positions of the resonance in air and in water. This can be understood by the fact that the cladding mode is partially exposed to both media, and it is expected that the relative amplitudes of the split resonances can be related to the fraction of the fiber circumference that is immersed. Consequently, when the fiber is pushed from the air into the water bead, the ST of the water acting on the TFBG will result in a larger reaction force against it, and an increasing contact angle which can be inferred from changes in the transmission spectrum of the TFBG. Given a calibration of the relative amplitudes of a resonance as a function of the contact angle, these spectral changes can be used to measure the force exerted by the bead on the TFBG if a relation between the contact angle and force can be determined.

Figure 3 illustrates how to calculate the restoring upward force on the TFBG as it is pushed against the water bead, from the ST and the contact angle. In static equilibrium, the restoring force must be equal to the downward force exerted by the fiber (rigidly fixed into the holder) on the bead. Neglecting end effects, since the length of contact between fiber and water is much larger than the fiber diameter, the upward force $F_{\text {up }}$ can be expressed as a function of ST and the contact angle $(\theta)$ as [23]

$$
F_{\text {up }}=2 F_{\text {ST }} \sin \theta=2 \mathrm{ST} L \sin \theta \text {, }
$$

where $L$ is the length of fiber in contact with the bead $(1 \mathrm{~cm}$ in our experiments). Given the surface tension of water at room temperature $(0.073 \mathrm{~N} / \mathrm{m})$, the maximum force observed when the contact angle approaches $90^{\circ}$ will be $1.46 \mathrm{mN}$. Another contribution to the upward force could come from buoyancy, i.e., a force equivalent to the weight of the displaced volume of water from the Archimedes principle. The approximate maximum value of this force should occur for a fully submerged fiber and, in this case, it will be equal to the density of water $\left(1 \mathrm{~g} / \mathrm{cm}^{3}\right)$ multiplied by the length of the fiber $(1 \mathrm{~cm})$ and by its cross section $\left(\pi r^{2}\right.$ where $\left.r=62.5 \mu \mathrm{m}\right)$. This calculation gives a value of $1.2 \mu \mathrm{N}$, i.e., three orders of magnitude smaller than the force due to $\mathrm{ST}$ and, thus, negligible.

Figure 4(a) shows the evolution of a polarized resonance of the TFBG (a resonance located near $1544 \mathrm{~nm}$ ) when its contact angle with water changes from $0^{\circ}$ to $90^{\circ}$. In this context, the
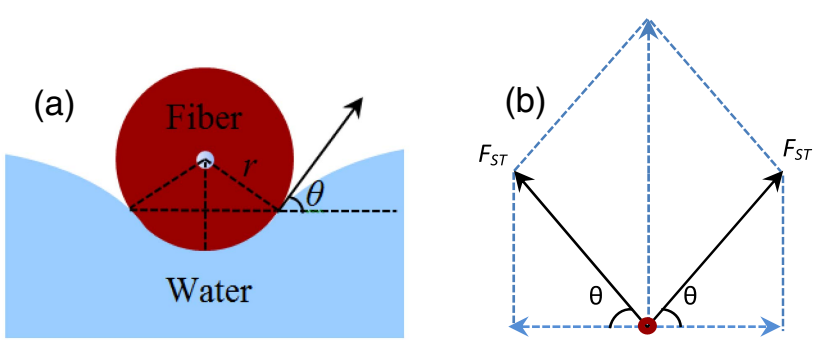

Fig. 3. Calculating the restoring upward force on the TFBG as it is pushed against the water bead. (a) Diagram of the TFBG on water. (b) Upward force on the TFBG analysis.
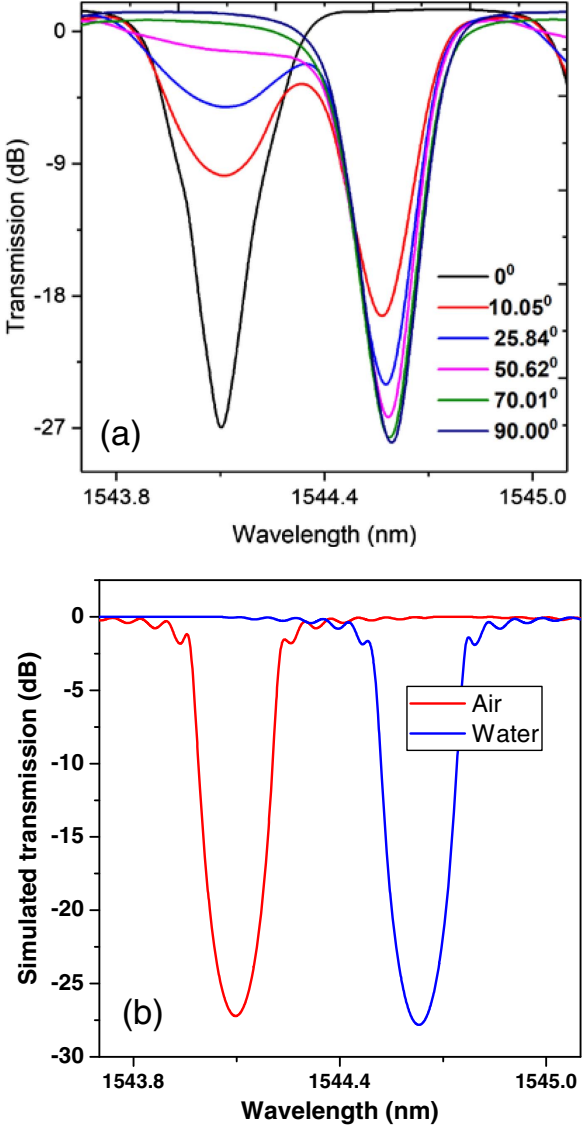

Fig. 4. (a) Transmission spectra of the TFBG near $1544 \mathrm{~nm}$ during the application of force on the surface of the water bead. The legend indicates the measured contact angle for each spectrum. (b) Simulation of the transmission spectra of the TFBG in air and in water near $1544 \mathrm{~nm}$.

increase in the contact angle means that the TFBG is brought in contact with the surface of water with increasing force. The contact angle for the last measurement where the TFBG is partially immersed was measured to be $70.01^{\circ}$. The fiber becomes fully immersed at the next data point $\left(90^{\circ}\right.$ angle). As shown in Fig. 4(b), we also made a simulation of the transmission spectra of the TFBG in air and in water, according to the coupled mode theory [24]. It can be seen that the experimental spectra are coincided with the simulation results.

It is clear from this experiment that as the water gradually surrounds the cladding surface, the surrounding medium changes from "air-like" (RI near 1.0) to "water-like" (RI near 1.315 at this wavelength) and, as a result, the resonance that is initially located at $1544.1 \mathrm{~nm}$ decreases in amplitude to be replaced by an increasing resonance at $1544.7 \mathrm{~nm}$. In order to establish a relationship between the applied force and the spectral changes, the force exerted by the fiber against the ST of the water for each spectrum can be calculated by Eq. (3). Then, as a quantitative measure of the spectral change, we used the relative recovery (RR) in the linear scale of the transmission (in decibels) of the resonance at $1544.1 \mathrm{~nm}$ relative to the transmission in water, defined as follows:

$$
\operatorname{RR}(\%)=100 \times 10^{\left(T(\theta)-T\left(90^{\circ}\right)\right) / 10} .
$$




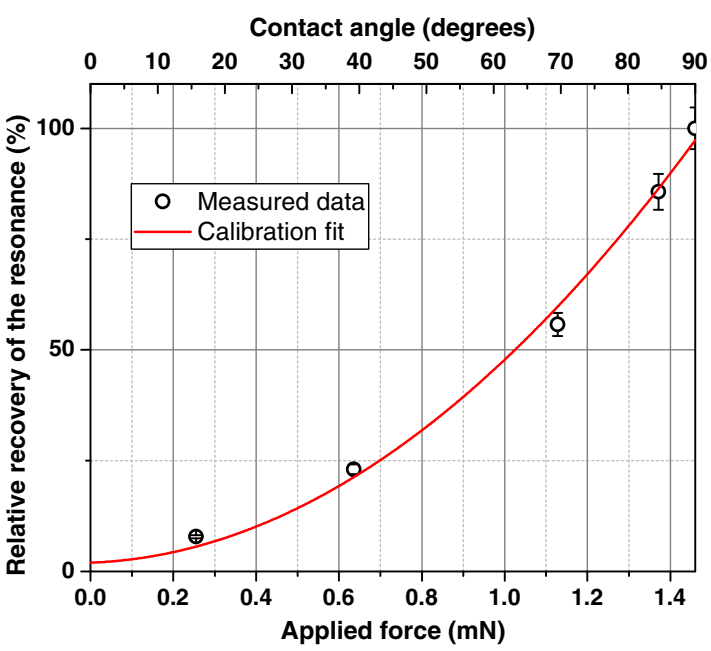

Fig. 5. Measured resonance loss as a function of applied force on the TFBG. The differences between the fit (calibration curve) correspond to errors ranging from 0.04 to $0.08 \mathrm{mN}$ of force.

The result of this analysis is plotted in Fig. 5 for values of RR as a function of both the contact angle and applied force. The measurements were carried out 10 times each and fell within the error bars shown. A second-order polynomial fit of the data yielded the following expression for RR in terms of applied force in milli-Newtons, with an R-squared value of 0.9932 :

$$
\mathrm{RR}=1.97+3.41 F+42.40 F^{2} \% .
$$

Vertical error bars on the data points correspond to the noise level of the transmission measurements. For the power fluctuation of the light source, we can use the measured transmission of the Bragg mode of the TFBG as a reference to cancel it (as the core mode is insensitive to the medium outside the cladding). If the fit curve is used as the calibration of the TFBG, the distribution of the data points away from the curve indicate that the applied force can be estimated from the spectral transmission change with an error inferior to $0.1 \mathrm{mN}$. It must be pointed out that this approach can be used to determine an unknown ST because what the RR parameter provides directly is the contact angle (more precisely, the fraction of the fiber circumference that is covered by the liquid), and Eq. (3) indicates that if a separate measurement of the force exerted on the TFBG can be made, then the value of the ST can be obtained.

In conclusion, the sensitivity of the cladding mode resonances of a TFBG to external RI has been used to measure lateral forces in the range of $0.2-1.4 \mathrm{mN}$, provided the applied force is exerted against a TFBG in contact with a liquid surface with a known ST. This was demonstrated with a $1 \mathrm{~cm}$ long TFBG pushed against the ST of a fixed bead of water, from the initial contact to the moment, where the force exceeds what the ST can support. Following calibration of the changes in the transmission, a TFBG at a given resonance wavelength against applied force (using the separately measured contact angle between the cladding surface and the liquid to calculate the force), any other force within the calibrated range can be measured with an accuracy of the order of $0.1 \mathrm{mN}$ from a simple spectral transmission measurement. We also indicate how to perform the complementary measurement, i.e., finding an unknown ST value from the TFBG spectral changes, provided a separate measurement of the contact angle can be made.

Funding. National Natural Science Foundation of China (NSFC) (61727816); China Scholarship Council (CSC) (201608330010); Natural Science Foundation of Zhejiang Province (LY16A040005); Special Fund for Quality Inspection Research in the Public Interest of China (201510066); National Key R\&D Program "National Quality Base Common Technology Research and Application" Key Special Projects of China (2017YFF0209703); Natural Sciences and Engineering Research Council of Canada (NSERC).

\section{REFERENCES}

1. S. Adera, R. Raj, R. Enright, and E. N. Wang, Nat. Commun. 4, 2518 (2013).

2. J.-S. Koh, E. Yang, G.-P. Jung, S.-P. Jung, J. H. Son, S.-I. Lee, P. G. Jablonski, R. J. Wood, H.-Y. Kim, and K.-J. Cho, Science 349, 517 (2015).

3. J. Meseguer, A. Sanz-Andrés, I. Pérez-Grande, S. Pindado, S. Franchini, and G. Alonso, Eur. J. Phys. 35, 055010 (2014).

4. L. Gao and T. J. McCarthy, Langmuir 22, 6234 (2006).

5. T. Deng, K. K. Varanasi, M. Hsu, N. Bhate, C. Keimel, J. Stein, and M. Blohm, Appl. Phys. Lett. 94, 133109 (2009).

6. H.-M. Kwon, A. T. Paxson, K. K. Varanasi, and N. A. Patankar, Phys. Rev. Lett. 106, 036102 (2011).

7. D.-H. Kwon and S.-J. Lee, Appl. Phys. Lett. 100, 171601 (2012).

8. A. Grigoryev, I. Tokarev, K. G. Kornev, I. Luzinov, and S. Minko, J. Am. Chem. Soc. 134, 12916 (2012).

9. A. Tuteja, W. Choi, M. Ma, J. M. Mabry, S. A. Mazzella, G. C. Rutledge, G. H. McKinley, and R. E. Cohen, Science 318, 1618 (2007).

10. I. U. Vakarelski, N. A. Patankar, J. O. Marston, D. Y. C. Chan, and S. T. Thoroddsen, Nature 489, 274 (2012).

11. L. Y. Shao and J. Albert, Opt. Commun. 284, 1855 (2011).

12. Y. Q. Liu, K. S. Chiang, and P. L. Chu, IEEE Photon. Technol. Lett. 17, 450 (2005).

13. B. Dong, D. P. Zhou, L. Wei, W. K. Liu, and J. W. Y. Lit, Opt. Express 16, 19291 (2008).

14. K. M. Chung, Z. Liu, C. Lu, and H. Y. Tam, IEEE Photon. Technol. Lett. 24, 700 (2012).

15. W. Luo, J. L. Kou, Y. Chen, F. Xu, and Y. Q. Lu, Appl. Phys. Lett. 101 133502 (2012).

16. T. Wieduwilt, S. Bruckner, and H. Bartelt, Meas. Sci. Technol. 22 075201 (2011).

17. Y. Gong, C. B. Yu, T. T. Wang, X.-P. Liu, Y. Wu, Y.-J. Rao, M.-L. Zhang, H.-J. Wu, X.-X. Chen, and G.-D. Peng, Opt. Express 22, 3578 (2014).

18. J. Albert, L. Y. Shao, and C. Caucheteur, Laser Photon. Rev. 7, 83 (2013).

19. C. Shen, L. Xiong, A. Bialiayeu, Y. Zhang, and J. Albert, J. Lightwave Technol. 32, 2157 (2014).

20. W. Zhou, D. J. Mandia, S. T. Barry, and J. Albert, Opt. Lett. 40, 1713 (2015).

21. Y. Wang, C. Shen, W. Lou, F. Shentu, C. Zhong, X. Dong, and L. Tong, Appl. Phys. Lett. 109, 031107 (2016).

22. C. Shen, Y. Zhang, W. Zhou, and J. Albert, Appl. Phys. Lett. 104, 071106 (2014).

23. D. L. Hu, B. Chan, and J. W. M. Bush, Nature 424, 663 (2003).

24. T. Ergogan and J. Sipe, J. Opt. Soc. Am. A13, 296 (1996). 